\title{
Twisted self-duality for higher spin gauge fields and prepotentials
}

\author{
Marc Henneaux, ${ }^{1}$ Sergio Hörtner, ${ }^{2}$ and Amaury Leonard ${ }^{1}$ \\ ${ }^{1}$ Université Libre de Bruxelles and International Solvay Institutes, ULB-Campus Plaine CP231, \\ B-1050 Brussels, Belgium \\ ${ }^{2}$ Centro de Estudios Científicos (CECs), Casilla 1469, Valdivia, Chile
}

(Received 2 October 2016; published 28 November 2016)

\begin{abstract}
We show that the equations of motion for (free) integer higher spin gauge fields can be formulated as twisted self-duality conditions on the higher spin curvatures of the spin-s field and its dual. We focus on the case of four spacetime dimensions, but formulate our results in a manner applicable to higher spacetime dimensions. The twisted self-duality conditions are redundant and we exhibit a nonredundant subset of conditions, which have the remarkable property to involve only first-order derivatives with respect to time. This nonredundant subset equates the electric field of the spin-s field (which we define) to the magnetic field of its dual (which we also define), and vice versa. The nonredundant subset of twisted self-duality conditions involve the purely spatial components of the spin-s field and its dual, and also the components of the fields with one zero index. One can get rid of these gauge components by taking the curl of the equations, which does not change their physical content. In this form, the twisted self-duality conditions can be derived from a variational principle that involves prepotentials. These prepotentials are the higher spin generalizations of the prepotentials previously found in the spins 2 and 3 cases. The prepotentials have again the intriguing feature of possessing both higher spin diffeomorphism invariance and higher spin conformal geometry. The tools introduced in an earlier paper for handling higher spin conformal geometry turn out to be crucial for streamlining the analysis. In four spacetime dimensions where the electric and magnetic fields are tensor fields of the same type, the twisted self-duality conditions enjoy an $S O(2)$ electric-magnetic invariance. We explicitly show that this symmetry is an "off-shell symmetry" (i.e., a symmetry of the action and not just of the equations of motion). Remarks on the extension to higher dimensions are given.
\end{abstract}

DOI: 10.1103/PhysRevD.94.105027

\section{INTRODUCTION}

Gravitational theories exhibit fascinating "hidden symmetries" upon dimensional reduction [1,2]. These hidden symmetries involve duality in an essential way. For instance, in the case of 11-dimensional supergravity reduced to four dimensions, the hidden $E_{7}$ symmetry includes $S O(2)$ electric-magnetic duality invariance acting on the 28 Abelian gauge fields present in the theory [2].

It has been conjectured that these hidden symmetries might actually already be present prior to dimensional reduction, although not manifestly so, and recent analysis in the light cone formalism supports this conjecture [3]. It has also been speculated that the finite-dimensional symmetries uncovered by dimensional reduction to $D \geq$ 3 dimensions are actually a subset of a much larger, infinite-dimensional Kac-Moody algebra, which could be $E_{10}$ or $E_{11}$ [4-10] for (an appropriate extension of) maximal supergravity.

Published by the American Physical Society under the terms of the Creative Commons Attribution 3.0 License. Further distribution of this work must maintain attribution to the author(s) and the published article's title, journal citation, and DOI.
A characteristic feature of the nonlinear realizations of the conjectured hidden symmetry algebras is that they treat the $p$-forms and their duals democratically: for each $p$-form appearing in the spectrum, the dual $D-p-2$ form also appears. In order to exhibit the hidden symmetries of gravitational theories, it appears therefore necessary to reformulate the equations of motion for the $p$-forms present in the model in a manner that involves both the $p$-forms and their duals on an equal footing, but without doubling the number of degrees of freedom. This is achieved by rewriting the equations of motion as "twisted self-duality conditions" $[2,11,12]$. These conditions are first-order with respect to time (and space) and equate the electric field (respectively the magnetic field) of the $p$-form to the magnetic field (respectively, \pm the electric field) of its dual. It is easily verified that these conditions are equivalent to the Maxwell equations. Furthermore, they can readily accommodate Chern-Simons couplings. This form of the equations of motion for the 3- and 7-forms of 11dimensional supergravity is the starting point of the authors of [13] in their recent construction of $E_{11}$-invariant equations of motion.

The twisted self-duality conditions derive from a variational principle where both the $p$-form potential and its dual are treated as independent fields on an equal footing [14], 
which is however not manifestly spacetime covariant (for comments on this fact, see [15]).

Now, the nonlinear realizations of all the conjectured infinite-dimensional hidden symmetries of gravitational theories involve also the dual to the graviton $[9,10]$. For that reason, similar twisted-duality reformulations of gravity are desirable. The task of rewriting the linearized gravitational field equations as twisted self-duality conditions putting the spin-2 field and its dual on a democratic footing, in a manner derivable from a variational principle, has been achieved in $[16,17]$.

One of the original motivations underlying the $E_{10}$ conjecture [10] was its potential connection with the zero tension limit of string theory [18]. This zero tension limit involves an infinite collection of massless higher spin fields. With this in mind, we continue in this paper our investigation of twisted self-duality for higher spin gauge fields. We consider explicitly the case of four spacetime dimensions but formulate our results in a manner applicable to higher spacetime dimensions [19].

We establish in this paper a number of results concerning the twisted self-duality formulation of higher spin gauge fields.

(i) We show that the equations of motion of the free bosonic higher spin fields can indeed equivalently be written as twisted self-duality conditions on the curvatures of the spin-s field and its dual (Sec. II). The crucial property that allows this reformulation is the demonstration given in [20] that the equations of motion of the higher spins are equivalent to the vanishing of the Ricci tensor. This is not obvious when $s>2$ since the equations of motion are of second order, while the curvatures contain derivatives of the higher spin gauge field up to the order $s$.

(ii) The twisted self-duality conditions are highly redundant. In Sec. III, we decompose the higher spin curvatures into electric and magnetic components, and point out that the subset of the twisted selfduality conditions that expresses that the electric field (respectively the magnetic field) of the spin- $s$ field is equal to the magnetic field (respectively, minus the electric field) of its dual, completely captures the full content of the twisted self-duality conditions. [The proof of this fact is postponed to Sec. V.] Remarkably, this subset of the twisted selfduality conditions contains only first-order time derivatives of the fields-even though a generic curvature component can contain up to $s$ time derivatives.

(iii) In their "electric-magnetic" form, the twisted selfduality conditions involve the spatial components of the spin $s$-field and its dual, as well as the components with one index in the time direction, i.e., equal to zero. These components are pure gauge and can be eliminated by taking an appropriate curl. The resulting equations are physically equivalent and shown to be derivable from a variational principle in Sec. IV. This variational principle naturally involves prepotentials, which are introduced to take into account the constraints on the electric and magnetic fields. The prepotentials enjoy spin- $s$ diffeomorphism invariance and also, somewhat unexpectedly, spin- $s$ Weyl invariance. The tools necessary to introduce the prepotentials have been developed in [21], upon which we heavily rely.

(iv) It turns out that the action principle so derived is exactly the action principle that one would obtain by starting from the Fronsdal action, going to the Hamiltonian formalism and solving the constraints through prepotentials. This is proved in Sec. V. As a by-product of this result follows the completeness of the twisted self-duality conditions on the electric and magnetic fields.

(v) Although the analysis is explicitly carried out in four spacetime dimensions, we expect that it should go through along parallel lines in higher dimensions where the higher spin field equations also admit a twisted self-duality reformulation. Arguments supporting this expectation, and how the analysis would proceed, are outlined in Sec. VI. There is, however, a feature peculiar to four dimensions (for the types of Young tableaux under consideration), namely, that the electric and magnetic fields are tensors of identical type, and the equations of motion are invariant under $S O(2)$ electric-magnetic duality rotations in the internal plane of the electric and magnetic fields. The action expressed in terms of the prepotentials makes it explicit that this symmetry is an off-shell symmetry. This result generalizes to higher spins the known results for spins $s=1$ [22], $s=2[23,24]$ and $s=3[21,25]$. This is also discussed in Sec. VI.

We conclude in Section VII with some comments. Two appendixes provide the technical steps necessary to derive the expression of the spin-s field in terms of the prepotentials.

This paper was announced in [21], with the different title "Emergent conformal geometry for higher spins."

\section{TWISTED SELF-DUALITY FOR HIGHER SPIN GAUGE FIELDS}

\section{A. Standard form of the equations of motion}

In four dimensions, a massless field of spin (helicity) $s$ is described by a completely symmetric tensor $h_{\lambda_{1} \lambda_{2} \cdots \lambda_{s}}$ of rank $s$, subject to the gauge invariance

$$
\delta h_{\lambda_{1} \lambda_{2} \cdots \lambda_{s}}=s \partial_{\left(\lambda_{1}\right.} \varepsilon_{\left.\lambda_{2} \cdots \lambda_{s}\right)}
$$

where the gauge parameter $\varepsilon_{\lambda_{2} \cdots \lambda_{s}}$ is completely symmetric but otherwise arbitrary. The gauge invariant curvatures involve $s$ derivatives of the fields and read [26] 
TWISTED SELF-DUALITY FOR HIGHER SPIN GAUGE ...

$$
R_{\lambda_{1} \mu_{1} \lambda_{2} \mu_{2} \cdots \lambda_{s} \mu_{s}}[h]=2^{s} \partial_{\left[\mu_{1} \mid\right.} \partial_{\left[\mu_{2} \mid\right.} \cdots \partial_{\left[\mu_{s} \mid\right.} h_{\left.\left.\left.\lambda_{1}\right] \mid \lambda_{2}\right] \mid \cdots \lambda_{s}\right]}
$$

It has Young symmetry type

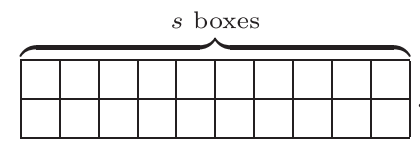

One can express it in terms of the derivative operator $d_{(s)}$ of [27,28], which fulfills $d_{(s)}^{s+1}=0$ (see also [29,30]). One has $R=d_{(s)}^{s} h$ and $\delta h=d_{(s)} \varepsilon$ (suppressing indices), so that $\delta R=d_{(s)}^{s+1} \varepsilon=0$. The curvature tensor fulfills also the "Bianchi identities"

$$
d_{(s)} R=0 .
$$

In order to get gauge invariant objects that involve no more than second order derivatives, it is necessary to restrict the gauge parameter $\varepsilon_{\lambda_{1} \cdots \lambda_{s-1}}$ to be traceless when $s>2$ [31]. The Fronsdal tensor [32],

$$
\begin{aligned}
F_{\lambda_{1} \cdots \lambda_{s}}= & \square h_{\lambda_{1} \cdots \lambda_{s}}-s \partial_{\left(\lambda_{1} \mid\right.} \partial^{\mu} h_{\left.\mu \mid \lambda_{2} \cdots \lambda_{s}\right)} \\
& +\frac{s(s-1)}{2} \partial_{\left(\lambda_{1}\right.} \partial_{\lambda_{2}} h_{\left.\lambda_{3} \cdots \lambda_{s}\right)}
\end{aligned}
$$

which involves only second order derivatives of the gauge field, is easily seen to be invariant when $\varepsilon_{\lambda_{1} \cdots \lambda_{s-1}}$ is traceless. In that approach where the gauge parameter is restricted by trace conditions, the equations of motion are actually

$$
F_{\lambda_{1} \cdots \lambda_{s}}=0
$$

and are derived from the Fronsdal action [32]

$$
\begin{aligned}
S\left[h_{\mu_{1} \mu_{2} \cdots \mu_{s}}\right]= & \int d^{4} x\left[-\frac{1}{2}\left(\partial_{\rho} h_{\mu_{1} \cdots \mu_{s}}\right)^{2}\right. \\
& +\frac{s}{2}\left(\partial^{\rho} h_{\rho \mu_{1} \cdots \mu_{s-1}}\right)^{2}-\left(\begin{array}{l}
s \\
2
\end{array}\right) \partial^{\rho} h_{\rho \sigma \mu_{1} \cdots \mu_{s-2}} \partial^{\sigma} \bar{h}^{\mu_{1} \cdots \mu_{s-2}} \\
& \left.+\frac{1}{2}\left(\begin{array}{l}
s \\
2
\end{array}\right)\left(\partial_{\rho} \bar{h}_{\mu_{1} \cdots \mu_{s-2}}\right)^{2}+\frac{3}{4}\left(\begin{array}{l}
s \\
3
\end{array}\right)\left(\partial^{\rho} \bar{h}_{\rho \mu_{1} \cdots \mu_{s-3}}\right)^{2}\right]
\end{aligned}
$$

(where $\bar{h}_{\mu_{1} \cdots \mu_{s-2}}=h_{\nu \mu_{1} \cdots \mu_{s-2}}^{\nu}$ ) which is easily verified to be gauge invariant up to a total derivative (with traceless gauge parameter).

\section{B. Equations in terms of the curvature}

In a very beautiful piece of work [20], it has been shown that the tracelessness condition on the gauge parameter is not necessary and can be viewed as a partial gauge condition. Equivalent ideas were formulated in [33], but their realization involves nonlocal terms, and for that reason we shall follow here [20].
PHYSICAL REVIEW D 94, 105027 (2016)

The equations of motion for a spin $s$ gauge field can be taken to be

$$
\bar{R}_{\lambda_{1} \lambda_{2} \lambda_{3} \mu_{3} \cdots \lambda_{s} \mu_{s}}=0
$$

where $\bar{R}$ is the "Ricci" tensor obtained by taking one trace on the Riemann tensor,

$$
\begin{aligned}
\bar{R}_{\lambda_{1} \lambda_{2} \lambda_{3} \mu_{3} \cdots \lambda_{s} \mu_{s}}= & 2^{s-2}\left\{\square \partial_{\left[\mu_{3} \mid\right.} \cdots \partial_{\left[\mu_{s} \mid\right.} h_{\left.\left.\lambda_{1} \lambda_{2} \mid \lambda_{3}\right] \cdots \lambda_{s}\right]}\right. \\
& -\partial_{\lambda_{1}} \partial^{\mu} \partial_{\left[\mu_{3} \mid\right.} \cdots \partial_{\left[\mu_{s}\right.} h_{\left.\left.\mu \lambda_{2} \mid \lambda_{3}\right] \mid \cdots \lambda_{s}\right]} \\
& \left.-\partial_{\lambda_{2}} \partial^{\mu} \partial_{\left[\mu_{3} \mid\right.} \cdots \partial_{\left[\mu_{s} \mid\right.} h_{\left.\left.\mu \lambda_{1} \mid \lambda_{3}\right] \mid \cdots \lambda_{s}\right]}\right] \\
& \left.\left.+\partial_{\lambda_{1}} \partial_{\lambda_{2}} \partial_{\left[\mu_{3} \mid\right.} \cdots \partial_{\left[\mu_{s} \mid\right.} \bar{h}_{\left.\lambda_{3}\right]}\right] \cdots \lambda_{s}\right]
\end{aligned}
$$

The equations (2.7) are differential equations of order $s$, but contrary to (2.5), they are invariant under the full gauge symmetry (2.1) without restriction on the trace of the gauge parameter. It was shown in [20] that they imply, with an appropriate choice of the trace of $\varepsilon_{\lambda_{1} \cdots \lambda_{s-1}}$, the Fronsdal equations (2.5) — which, conversely, are easily verified to imply (2.7).

As also pointed out in [20], the equations (2.7) are very convenient for discussing duality along the lines of [34]. Let $S_{\lambda_{1} \mu_{1} \lambda_{2} \mu_{2} \cdots \lambda_{s} \mu_{s}}$ be the tensor dual to $R_{\lambda_{1} \mu_{1} \lambda_{2} \mu_{2} \cdots \lambda_{s} \mu_{s}}$ on the first two indices (say),

$$
\begin{aligned}
S_{\lambda_{1} \mu_{1} \lambda_{2} \mu_{2} \cdots \lambda_{s} \mu_{s}} & ={ }^{*} R_{\lambda_{1} \mu_{1} \lambda_{2} \mu_{2} \cdots \lambda_{s} \mu_{s}} \\
& =\frac{1}{2} \epsilon_{\lambda_{1} \mu_{1}}^{\rho_{1} \sigma_{1}} R_{\rho_{1} \sigma_{1} \lambda_{2} \mu_{2} \cdots \lambda_{s} \mu_{s}}
\end{aligned}
$$

The equations of motion (2.7) imply that $S_{\lambda_{1} \mu_{1} \lambda_{2} \mu_{2} \cdots \lambda_{s} \mu_{s}}$ fulfills the cyclic identity, i.e., is a tensor of same Young type as $R_{\lambda_{1} \mu_{1} \lambda_{2} \mu_{2} \cdots \lambda_{s} \mu_{s}}$. Furthermore, the cyclic identity for $R_{\lambda_{1} \mu_{1} \lambda_{2} \mu_{2} \cdots \lambda_{s} \mu_{s}}$ implies that $S_{\lambda_{1} \mu_{1} \lambda_{2} \mu_{2} \cdots \lambda_{s} \mu_{s}}$ is traceless, $\bar{S}_{\lambda_{1} \lambda_{2} \lambda_{3} \mu_{3} \cdots \lambda_{s} \mu_{s}}=0$. There is thus complete symmetry between the equations fulfilled by $R$ and its dual $S$.

\section{Twisted self-duality}

It is this symmetry which is embodied in the twisted selfduality formulation. When the equations of motion for the spin- $s$ field are fulfilled, the tensor $S$ dual to the curvature not only is of same Young symmetry type

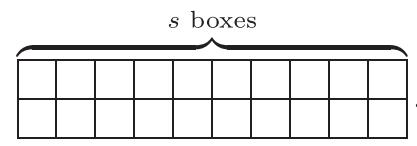

as $R$, but it fulfills also the Bianchi identities $d_{(s)} S=0$. This implies the existence of a "dual" spin- $s$ field $f_{\lambda_{1} \lambda_{2} \ldots \lambda_{s}}$ of which $S$ is the curvature [27,28],

$$
S=d_{(s)}^{s} f
$$

This second spin-s field has its own gauge invariance since it is determined up to the $d_{(s)}$ of some $\eta_{\lambda_{1} \cdots \lambda_{s-1}}$, 


$$
\delta f_{\lambda_{1} \lambda_{2} \cdots \lambda_{s}}=s \partial_{\left(\lambda_{1}\right.} \eta_{\left.\lambda_{2} \cdots \lambda_{s}\right)}
$$

We can thus rewrite the equations of motion for the spin$s$ theory in a duality-symmetric way where both the spin-s field and its dual appear on an equal footing as follows,

$$
\mathcal{F}=\mathcal{S}^{*} \mathcal{F},
$$

where,

$\mathcal{F}=\left(\begin{array}{c}R[h] \\ S[f]\end{array}\right), \quad * \mathcal{F}=\left(\begin{array}{c}* R[h] \\ * S[f]\end{array}\right), \quad \mathcal{S}=\left(\begin{array}{cc}0 & -1 \\ 1 & 0\end{array}\right)$.

This form of the equations is completely equivalent to the original form $\bar{R}=0$, since we have seen that the equation $\bar{R}=0$ implies (2.11). And conversely, if (2.11) holds, then both $h$ and $f$ obey $\bar{R}[h]=0, \bar{S}[f]=0$, i.e., fulfill the spin- $s$ equations of motion. Furthermore, the two spin-s fields are not independent since $f$ is completely determined by $h$ up to a gauge transformation and therefore carries no independent physical degrees of freedom.

Following [12], one refers to (2.11) as the twisted selfdual formulation of the spin-s theory.

\section{ELECTRIC AND MAGNETIC FIELDS}

\section{A. Definitions}

The twisted self-duality conditions in their covariant form (2.11) are highly redundant. We shall extract from them an equivalent subset that has the interesting property of containing only first order derivatives with respect to time.

To that end, we first define the electric and magnetic components of the Weyl tensor, which coincides on-shell with the Riemann tensor. It would seem natural to define the electric components as the components of the Weyl tensor with the maximum number of indices equal to zero (namely $s$ ), and the magnetic components as the components with the maximum number minus one of indices equal to zero (namely $s-1$ ). By the tracelessness conditions of the Weyl tensor, the electric components can be related to the components with no zeroes when $s$ is even, like for gravity, or just one zero when $s$ is odd, like for Maxwell. It turns out to be more convenient for dynamical purposes to define the electric and magnetic components starting from the other end, i.e., in terms of components with one or no zero. Now, it would be cumbersome in the general analysis to have a definition of the electric and magnetic components that would depend on the spin. For that reason, we shall adopt a definition which is uniform for all spins, but which coincides with the standard conventions given above only for even spins. It makes the Schwarzschild field "electric," but the standard electric field of electromagnetism is viewed as "magnetic." Since the electric (magnetic) components of the curvature of the spin-s field are the magnetic (electric) components of the curvature of the dual spin-s field, this is just a matter of convention, but this convention may be confusing when confronted with the standard Maxwell terminology.

Before providing definitions, we recall that the curvature $R_{i_{1} j_{1} \cdots i_{s} j_{s}}$ of the three-dimensional "spin-s field" $h_{i_{1} \cdots i_{s}}$ given by the spatial components of the spacetime spin-s field $h_{\lambda_{1} \cdots \lambda_{s}}$ is completely equivalent to its Einstein tensor defined as

$$
G^{i_{1} \cdots i_{s}}=\frac{1}{2^{s}} \epsilon^{i_{1} j_{1} k_{1}} \cdots \epsilon^{i_{s} j_{s} k_{s}} R_{j_{1} k_{1} \cdots j_{s} k_{s}} .
$$

This tensor is completely symmetric and identically conserved,

$$
\partial_{i_{1}} G^{i_{1} i_{2} \cdots i_{s}}=0 .
$$

In the sequel, when we shall refer to the Einstein tensor of the spin $s$ field, we shall usually mean this threedimensional Einstein tensor (the four-dimensional Einstein tensor vanishes on-shell).

We now define precisely the electric and magnetic fields off-shell as follows:

(i) The electric field $\mathcal{E}^{i_{1} \cdots i_{s}}$ of the spin-s field $h_{\lambda_{1} \cdots \lambda_{s}}$ is equal to the Einstein tensor $G^{i_{1} \cdots i_{s}}$ of its spatial components $h_{i_{1} \cdots i_{s}}$,

$$
\mathcal{E}^{i_{1} \cdots i_{s}}=G^{i_{1} \cdots i_{s}} .
$$

By construction, the electric field fully captures the spatial curvature and involves only the spatial components of the spin-s field. It is completely symmetric and conserved,

$$
\mathcal{E}^{i_{1} \cdots i_{s}}=\mathcal{E}^{\left(i_{1} \cdots i_{s}\right)}, \quad \partial_{i_{1}} \mathcal{E}^{i_{1} i_{2} \cdots i_{s}}=0 .
$$

(ii) The magnetic field $\mathcal{B}^{i_{1} \cdots i_{s}}$ of the spin-s field $h_{\lambda_{1} \cdots \lambda_{s}}$ is equivalent to the components with one zero of the spacetime curvature tensor and is defined through

$$
\mathcal{B}_{i_{1} \cdots i_{s}}=\frac{1}{2^{s-1}} R_{0 i_{1}}^{j_{2} k_{2} \cdots j_{s} k_{s}} \epsilon_{i_{2} j_{2} k_{2}} \cdots \epsilon_{i_{s} j_{s} k_{s}}
$$

It contains one time (and $s-1$ space) derivatives of the spatial components $h_{i_{1} \cdots i_{s}}$, and $s$ derivatives of the mixed components $h_{0 i_{2} \cdots i_{s}}$. The magnetic field is symmetric in its last $s-1$ indices. It is also transverse on each index,

$$
\partial_{i_{1}} \mathcal{B}^{i_{1} i_{2} \cdots i_{s}}=0, \quad \partial_{i_{2}} \mathcal{B}^{i_{1} i_{2} \cdots i_{s}}=0,
$$

and traceless on the first index and any other index,

$$
\delta_{i_{1} i_{2}} \mathcal{B}^{i_{1} i_{2} \cdots i_{s}}=0 .
$$


It is useful to make explicit the dependence of the magnetic field-or equivalently, $R_{0 i_{1} j_{2} k_{2} \cdots j_{s} k_{s}}$-on $h_{0 i_{2} \cdots i_{s}}$. One finds

$$
R_{0 i_{1} j_{2} k_{2} \cdots j_{s} k_{s}}=\partial_{i_{1}}\left(d_{(s-1)}^{s-1} N\right)_{j_{2} k_{2} \cdots j_{s} k_{s}}+\text { "more" }
$$

where "more" involves only spatial derivatives of $\dot{h}_{i_{1} \cdots i_{s}}$ and where $N_{i_{1} \cdots i_{s-1}}$ stands for $h_{0 i_{1} \cdots i_{s-1}}$, i.e., $N_{i_{1} \cdots i_{s-1}} \equiv h_{0 i_{1} \cdots i_{s-1}}$.

Similar definitions apply to the dual spin-s field $f_{\lambda_{1} \cdots \lambda_{s}}$.

The electric and magnetic fields possess additional properties on-shell. First, the electric field is traceless as a result of the equation $\bar{R}_{0 i_{5} \cdots i_{s}}^{0}-\frac{1}{2} \delta_{0}^{0} \overline{\bar{R}}_{i_{5} \cdots i_{s}}=0$,

$$
\delta_{i_{1} i_{2}} \mathcal{E}^{i_{1} i_{2} \cdots i_{s}}=0 .
$$

Second, the magnetic field is symmetric as a result of the equation $\bar{R}_{0 i_{4} i_{5} \cdots i_{s}}=0$,

$$
\mathcal{B}^{i_{1} \cdots i_{s}}=\mathcal{B}^{\left(i_{1} \cdots i_{s}\right)}=0 .
$$

We also note that there are no other independent components of the spacetime curvature tensor on-shell, since components with more than one zero can be expressed in terms of components with one or no zero through the equations of motion.

\section{B. Twisted self-duality in terms of electric and magnetic fields}

It is clear that the twisted self-duality conditions (2.11) with all indices being taken to be spatial read

$$
\left(\begin{array}{c}
\mathcal{E}^{i_{1} i_{2} \cdots i_{s}}[h] \\
\mathcal{E}^{i_{1} i_{2} \cdots i_{s}}[f]
\end{array}\right)=\left(\begin{array}{c}
\mathcal{B}^{i_{1} i_{2} \cdots i_{s}}[f] \\
-\mathcal{B}^{i_{1} i_{2} \cdots i_{s}}[h]
\end{array}\right)
$$

It turns out that these equations are completely equivalent to the full set of twisted self-duality conditions. This is not surprising since the components of the curvature tensor with two or more zeroes are not independent on-shell from the components with one or no zero. The fact that (3.11) completely captures all the equations of motion will be an automatic consequence of our subsequent analysis and so we postpone its proof to later (Sec. V below).

\section{Getting rid of the Lagrange multipliers}

While a generic component of the curvature may contain up to $s$ time derivatives, the twisted self-duality conditions (3.11) contain only the first-order time derivatives $\dot{h}_{i_{1} \cdots i_{s}}$ and $\dot{f}_{i_{1} \cdots i_{s}}$. One can give the fields $h_{i_{1} \cdots i_{s}}$ and $f_{i_{1} \cdots i_{s}}$ as Cauchy data on the spacelike hypersurface $x^{0}=0$. The subsequent values of these fields are determined by the twisted self-duality conditions up to gauge ambiguities. The Cauchy data $h_{i_{1} \cdots i_{s}}$ and $f_{i_{1} \cdots i_{s}}$ cannot be taken arbitrarily but must be such that their respective electric fields are both traceless since this follows from $\mathcal{E}= \pm \mathcal{B}$ and the fact that the magnetic field is traceless. The constraints are equivalent to the condition that the traces of the Einstein tensors of both $h$ and $f$ should be zero,

$$
\bar{G}^{i_{1} \cdots i_{s-2}}[h]=0, \quad \bar{G}^{i_{1} \cdots i_{s-2}}[f]=0 .
$$

The twisted self-duality conditions involve also the mixed components $h_{0 i_{2} \cdots i_{s}}$ and $f_{0 i_{2} \cdots i_{s}}$. These are pure gauge variables, which act as Lagrange multipliers for constraints in the Hamiltonian formalism. It is useful for the subsequent discussion to get rid of them. Since they occur only in the magnetic fields, and through a gradient, this can be achieved by simply taking a curl on the first index. Explicitly, from the twisted self-duality conditions (3.11) rewritten as

$$
\mathcal{E}^{a i_{1} \cdots i_{s}}=\epsilon^{a}{ }_{b} \mathcal{B}^{b i_{1} \cdots i_{s}}
$$

$\left(\mathcal{E}^{a i_{1} \cdots i_{s}} \equiv \mathcal{E}^{i_{1} \cdots i_{s}}\left[h^{a}\right], \quad \mathcal{B}^{a i_{1} \cdots i_{s}} \equiv \mathcal{B}^{i_{1} \cdots i_{s}}\left[h^{a}\right], \quad a=1, \quad 2\right.$, $\left.\left(h^{a}\right)=(h, f), \epsilon_{a b}=-\epsilon_{b a}, \epsilon_{12}=1\right)$, follows obviously the equation

$$
\epsilon_{j k i_{1}} \partial^{k} \mathcal{E}^{a i_{1} \cdots i_{s}}=\epsilon_{b}^{a} \epsilon_{j k i_{1}} \partial^{k} \mathcal{B}^{b i_{1} \cdots i_{s}}
$$

which does not involve the mixed components $h_{0 i_{2} \cdots i_{s}}$ or $f_{0 i_{2} \cdots i_{s}}$ any more.

The equations (3.14) are physically completely equivalent to (3.13). Indeed, it follows from (3.14) that

$$
\mathcal{E}^{a i_{1} \cdots i_{s}}=\epsilon_{b}^{a} \tilde{\mathcal{B}}^{b i_{1} \cdots i_{s}}
$$

where $\tilde{\mathcal{B}}^{b i_{1} \cdots i_{s}}$ differs from the true magnetic field $\mathcal{B}^{b i_{1} \cdots i_{s}}$ by an arbitrary gradient in $i_{1}$, or, in terms of the corresponding curvature components

$$
\tilde{R}_{0 i_{1} j_{2} k_{2} \cdots j_{s} k_{s}}^{a}=R_{0 i_{1} j_{2} k_{2} \cdots j_{s} k_{s}}^{a}+\partial_{i_{1}} \mu_{j_{2} k_{2} \cdots j_{s} k_{s}}^{a}
$$

for some arbitrary $\mu_{j_{2} k_{2} \cdots j_{s} k_{s}}^{a}$ with Young symmetry type

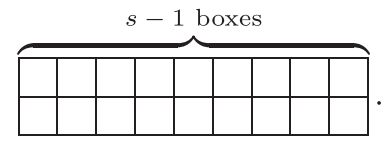

Now, the cyclic identity fulfilled by the curvature implies $\partial_{\left[i_{1}\right.} \mu_{\left.j_{2} k_{2}\right] \cdots j_{s} k_{s}}^{a}=0$, i.e., in index-free notation, $d_{(s-1)} \mu^{a}=0$, and this yields $\mu^{a}=d_{(s-1)}^{s-1} \nu^{a}$ for some symmetric $\nu_{j_{2} \cdots j_{s}}^{a}$ $[27,28]$. Comparing with $(3.8)$, we see that this is just the ambiguity in $R_{0 i_{1} j_{2} k_{2} \cdots j_{s} k_{s}}^{a}$ due to the presence of $h_{0 j_{2} \cdots j_{s}}^{a}$. Therefore, one can absorb $\mu_{j_{2} k_{2} \cdots j_{s} k_{s}}^{a}$ in a redefinition of the pure gauge variables $h_{0 j_{2} \cdots j_{s}}^{a}$ and get thereby Eqs. (3.13).

It is in the form (3.14) that we shall derive the twisted self-duality conditions from a variational principle. 


\section{VARIATIONAL PRINCIPLE}

\section{A. Prepotentials}

The searched-for variational principle involves as basic dynamical variables not the fields $h_{i_{1} \ldots i_{s}}^{a}$, which are constrained, but rather "prepotentials" that solve the constraints (3.12) and can be varied freely in the action. The general solution of the constraint equation $\bar{G}^{a i_{1} \cdots i_{s-2}}=0$ was worked out in [21] and implies the existence of prepotentials $Z_{i_{1} \cdots i_{s}}^{a}$ from which $h_{i_{1} \cdots i_{s}}^{a}$ derives, such that the Einstein tensor $G^{a} i_{1} \cdots i_{s}$ of $h_{i_{1} \cdots i_{s}}^{a}$ is equal to the Cotton tensor $D^{a i_{1} \cdots i_{s}}$ of $Z_{i_{1} \cdots i_{s}}^{a}$.

The Cotton tensor $D^{a i_{1} \cdots i_{s}}$ involves $2 s-1$ derivatives of the prepotentials and possesses the property of being invariant under spin-s diffeomorphisms and Weyl symmetries,

$$
\delta Z_{i_{1} \cdots i_{s}}^{a}=s \partial_{\left(i_{1}\right.} \rho_{\left.i_{2} \cdots i_{s}\right)}^{a}+\frac{s(s-1)}{2} \delta_{\left(i_{1} i_{2}\right.} \sigma_{\left.i_{3} \cdots i_{s}\right)}^{a} .
$$

It is symmetric, transverse and traceless. It was introduced for general spins in [35,36] and [21] (where it was denoted $B)$, and used extensively in three-dimensional higher spin models in [37-43].

Explicitly, the Cotton tensor of $Z_{i_{1} \cdots i_{s}}^{a}$ is given by

$$
\begin{aligned}
D^{a i_{1} i_{2} \cdots i_{s}}[Z]= & \varepsilon^{i_{1} j_{1} k_{1}} \varepsilon^{i_{2} j_{2} k_{2}} \cdots \varepsilon^{i_{s-1} j_{s-1} k_{s-1}} \\
& \times \partial_{j_{1}} \partial_{j_{2}} \cdots \partial_{j_{s-1}} S_{k_{1} k_{2} \cdots k_{s-1}}^{a}{ }^{i_{s}}[Z]
\end{aligned}
$$

where $S^{a i_{1} \cdots i_{s}}[Z]$ is the Schouten tensor of $Z_{i_{1} \cdots i_{s}}^{a}$, related to the Einstein tensor of $Z_{i_{1} \cdots i_{s}}^{a}$ through

$$
\begin{aligned}
S^{a i_{1} \cdots i_{s}}[Z]= & G^{a i_{1} \cdots i_{s}}[Z] \\
& +\sum_{n=1}^{\left[\frac{n}{2}\right]} c_{n} \delta^{\left(i_{1} i_{2}\right.} \cdots \delta^{i_{2 n-1} i_{2 n}} G_{[n]}^{\left.a i_{2 n+1} \cdots i_{s}\right)}[Z]
\end{aligned}
$$

with

$$
c_{n}=\frac{(-1)^{n}}{4^{n}} \frac{s}{n !} \frac{(s-n-1) !}{(s-2 n) !}, \quad(n \geq 1)
$$

(see [21]).

Because of the gauge symmetries, the solution of the equation $G^{a i_{1} \cdots i_{s}}[h]=D^{a i_{1} \cdots i_{s}}[Z]$ for $h_{i_{1} \cdots i_{s}}^{a}$ involves ambiguities. To any given solution $h^{a}[Z]$ one can add an arbitrary variation of $h_{i_{1} \cdots i_{s}}^{a}$ under spin-s diffeomorphisms. Furthermore $Z_{i_{1} \cdots i_{s}}^{a}$ and $Z_{i_{1} \cdots i_{s}}^{a}+\delta Z_{i_{1} \cdots i_{s}}^{a} \quad$ [with $\delta Z_{i_{1} \cdots i_{s}}^{a}$ given by (4.1)] yield $h^{a}[Z]$ 's that differ by a spin-s diffeomorphism.

The expression for the spin- $s$ field $h_{i_{1} \cdots i_{s}}$ in terms of the prepotential $Z_{i_{1} \cdots i_{s}}$ contains $s-1$ derivatives in order to match the number of derivatives $(s)$ of the Einstein tensor $G[h]$ with the number of derivatives $(2 s-1)$ of the Cotton tensor $D[Z]$. This number is odd (even) when $s$ is even (odd) and therefore, in order to match the indices of $h_{i_{1} \cdots i_{s}}$ with those of $\partial_{k_{1}} \cdots \partial_{k_{s-1}} Z_{j_{1} \cdots j_{s}}$, one needs one $\epsilon^{i j k}$ when $s$ is even and no $\epsilon^{i j k}$ when it is odd, together with products of $\delta_{i j}$ 's.

\section{Even spins}

We first turn to the even $s$ case. We recall that in the spin2 case, a particular solution is given by [23]

$$
h_{i j}=\epsilon_{(i \mid k l} \partial^{k} Z_{\mid j)}^{l}
$$

where the indices between the symbol || are omitted in the symmetrization - which is as usual carried with weight one such that it is a projector. The gauge freedom of the prepotential is given by

$$
\delta Z_{i j}=\delta_{i j} \sigma+2 \partial_{(i} \rho_{j)},
$$

which generates the particular diffeomorphism $\delta h_{i j}=$ $\partial_{(i} \theta_{j)}$ of the field, where $\theta_{i}=\epsilon_{i k l} \partial^{k} \rho^{l}$ (it is a diffeomorphism whose parameter is divergenceless). The generalization of this formula to general even spin $s=2 n$ is given in Appendix A.

We give here for definiteness the expression of the spin 4 field $h_{i j k l}$ in terms of its prepotential $\phi_{i j k l}$. One has

$$
\begin{aligned}
h_{i j k l}= & \epsilon_{(i \mid m n} \partial^{m}\left[-\Delta Z^{n}{ }_{\mid j k l)}+\frac{1}{2} \delta_{\mid j k} \Delta \bar{Z}^{n}{ }_{l)}\right. \\
& \left.-\frac{1}{2} \delta_{\mid j k} \partial^{p} \partial^{q} Z^{n}{ }_{l) p q}\right]
\end{aligned}
$$

The gauge freedom of the prepotential is given by:

$$
\delta Z_{i j k l}=4 \partial_{(i} \rho_{j k l)}+6 \delta_{(i j} \sigma_{k l)},
$$

which implies:

$$
\delta h_{i j k l}=\partial_{(i} \theta_{j k l)},
$$

where

$$
\begin{gathered}
\theta_{i j k}=\epsilon_{(i \mid m n} \partial^{m} \mu^{n}{ }_{\mid j k)}, \\
\mu_{i j k}=-3 \Delta \rho_{i j k}+\frac{1}{2} \delta_{(i j}\left[\Delta \bar{\rho}_{k)}-\partial^{p} \partial^{q} \rho_{k) p q}\right. \\
\left.-4 \partial^{p} \sigma_{k) p}\right] .
\end{gathered}
$$

In fact, as discussed in Appendix A, the expression (4.4) is, up to a multiplicative factor, the only one (with the requested number of derivatives) that implies that a gauge variation of $Z$ gives a gauge variation of $h$. 


\section{Odd spins}

In the odd spin case, the number of derivatives on the prepotential is even, so that the expression relating $h$ to $Z$ does not involve the Levi-Civita tensor. The expression for the spin-3 field in terms of its prepotential is explicitly given in [21]

$$
\begin{aligned}
h_{i j k}= & -\Delta Z_{i j k}+\frac{3}{4} \delta_{(i j} \Delta \bar{Z}_{k)} \\
& -\frac{3}{4} \delta_{(i j} \partial^{r} \partial^{s} Z_{k) r s}+\frac{3}{10} \delta_{(i j} \partial_{k)} \partial^{r} \bar{Z}_{r} .
\end{aligned}
$$

The last term in (4.9) is actually not necessary but included so that $\delta h_{i j k}=0$ under Weyl transformations of $Z$. One easily verifies that a gauge transformation of the prepotential induces a gauge transformation of the spin-3 field.

The expression of $h_{i_{1} \cdots i_{s}}$ in terms of $Z_{i_{1} \cdots i_{s}}$ is given in Appendix B for general odd spin.

\section{B. Twisted self-duality and prepotentials}

In terms of the prepotentials, the electric fields are given by

$$
\mathcal{E}^{a i_{1} \cdots i_{s}}=D^{a i_{1} \cdots i_{s}}[Z]
$$

while the magnetic fields have the property

$$
\epsilon_{j}{ }^{i_{1} k} \partial_{k} \mathcal{B}^{a j i_{2} \cdots i_{s}}=\dot{D}^{a i_{1} \cdots i_{s}}[Z] .
$$

It follows that the twisted self-duality conditions take the form

$$
\epsilon^{i_{1}}{ }_{j k} \partial^{j} D^{a k i_{2} \cdots i_{s}}[Z]=\epsilon^{a}{ }_{b} \dot{D}^{b i_{1} \cdots i_{s}}[Z]
$$

in terms of the prepotentials: the curl of the Cotton tensor of one prepotential is equal to $( \pm)$ the time derivative of the other.

\section{Action}

In their form (4.12), the twisted self-duality conditions are easily checked to derive from the following variational principle,

$$
S[Z]=\int d x^{0}\left[\int d^{3} x \frac{1}{2} \varepsilon_{a b} D^{a i_{1} \cdots i_{s}} \dot{Z}_{i_{1} \cdots i_{s}}^{b}-H\right]
$$

where the Hamitonian $H$ reads

$$
H=\int d^{3} x \delta_{a b}\left(\sum_{k=0}^{\left[\frac{s}{2}\right]} a_{k} G^{[k] a i_{1} \cdots i_{s-2 k}} G^{[k] b}{ }_{i_{1} \cdots i_{s-2 k}}\right)
$$

where $G^{[k] a i_{1} \cdots i_{s-2 n}}$ stands for the $k$ th trace of the Einstein tensor $G^{a i_{1} \cdots i_{s}}[Z]$ of the prepotential $Z_{i_{1} \cdots i_{s}}^{a}$. A lengthy but conceptually direct computation shows that the coefficients $a_{k}$ are explicitly given by

$$
a_{k}=(-)^{k} \frac{n !}{(n-k) ! k !} \frac{(2 n-k-1) !(2 n-1) ! !}{2^{k}(2 n-1) !(2 n-2 k-1) ! !} \frac{1}{2}
$$

for even spin $s=2 n$, and

$$
a_{k}=(-)^{k} \frac{n !}{(n-k) ! k !} \frac{(2 n-k) !(2 n+1) ! !}{2^{k}(2 n) !(2 n-2 k+1) ! !} \frac{1}{2}
$$

for odd spin $s=2 n+1$, where the definition of the double factorial is recalled in the Appendix. These coefficients are in fact uniquely determined up to an overall factor by the property that the action is invariant, up to a surface term, under the gauge symmetries (4.1) of the prepotentials. Invariance under spin-s diffeomorphisms is manifest, while invariance under spin-s Weyl symmetry forces $a_{k}$ to be given by the above expression (up to an overall factor).

\section{HAMILTONIAN FORMALISM}

\section{A. Constraints and Hamiltonian}

It turns out that the action (4.13) is exactly the action that one obtains by rewriting the Fronsdal action in Hamiltonian form and solving the constraints.

The procedure to establish this fact proceeds as follows.

(i) First one writes the Fronsdal action in Hamiltonian form $[44,45]$. The Hamiltonian canonical variables are the spatial components $h_{i_{1} \cdots i_{s}}$ of the spin-s field, their conjugate momenta $\pi^{i_{1} \cdots i_{s}}$, the variables $\alpha_{i_{1} \cdots i_{s-3}}$ equal to $h_{000 i_{1} \cdots i_{s-3}}-3 \delta^{k l} h_{0 i_{1} \cdots i_{s-3} k l}$ and their conjugate momenta $\tilde{\Pi}^{i_{1} \cdots i_{s-3}}$. The canonical action takes the form

$$
\begin{aligned}
& S\left[h_{i_{1} \cdots i_{s}}, \pi^{i_{1} \cdots i_{s}}, \alpha_{i_{1} \cdots i_{s-3}}, \tilde{\Pi}^{i_{1} \cdots i_{s-3}}, \mathcal{N}^{i_{1} \cdots i_{s-2}}, N^{i_{1} \cdots i_{s-1}}\right] \\
& =\int d x^{0}\left[\int d^{3} x\left(\pi^{i_{1} \cdots i_{s}} \dot{h}_{i_{1} \cdots i_{s}}+\tilde{\Pi}^{i_{1} \cdots i_{s-3}} \dot{\alpha}_{i_{1} \cdots i_{s-3}}\right)\right. \\
& \quad-H-\int d^{3} x\left(\mathcal{N}^{i_{1} \cdots i_{s-2}} \mathcal{C}_{i_{1} \cdots i_{s-2}}\right. \\
& \left.\left.\quad+N^{i_{1} \cdots i_{s-1}} \mathcal{C}_{i_{1} \cdots i_{s-1}}\right)\right]
\end{aligned}
$$

where $\mathcal{C}_{i_{1} \cdots i_{s-2}}$ and $\mathcal{C}_{i_{1} \cdots i_{s-1}}$ are the constraint-generators related to temporal $\left(\epsilon_{0 i_{1} \cdots i_{s-2}}\right)$ and spacelike $\left(\epsilon_{i_{1} \cdots i_{s-1}}\right)$ spin-s diffeomorphisms, respectively, and $\mathcal{N}_{i_{1} \cdots i_{s-2}}=h_{00 i_{1} \cdots i_{s-2}}$ and $N_{i_{1} \cdots i_{s-1}}=h_{0 i_{1} \cdots i_{s-1}}$ are the corresponding Lagrange multipliers. The explicit form of the constraints is rather cumbersome and has been given in [45]. The function $H$ is the Hamiltonian. It is the integral over space of a density $\mathcal{H}$ which is quadratic in the conjugate momenta and in the first spatial derivatives of $h_{i_{1} \cdots i_{s}}, \alpha_{i_{1} \cdots i_{s-3}}$, $H=\int d^{3} x \mathcal{H}$. We shall not need here the explicit expression of $\mathcal{H}$ in terms of $\partial_{k} h_{i_{1} \cdots i_{s}}, \pi^{i_{1} \cdots i_{s}}$, $\partial_{k} \alpha_{i_{1} \cdots i_{s-3}}, \tilde{\Pi}^{i_{1} \cdots i_{s-3}}$, which is also cumbersome. By 
analogy with the spin-2 case, we shall call the constraint

$$
\mathcal{C}_{i_{1} \cdots i_{s-2}}=0
$$

the "Hamiltonian constraint" and the constraint

$$
\mathcal{C}_{i_{1} \cdots i_{s-1}}=0
$$

the "momentum constraint."

(ii) The second step is to solve the constraints in terms of prepotentials $Z_{i_{1} \ldots i_{s}}^{a}, s=1,2$. One needs two prepotentials, one for solving the Hamiltonian constraint, the other one for solving the momentum constraint. The procedure uses the conformal tools developed in [21] and follows exactly the same pattern as for spins 2 and 3. It is displayed in the next two sections.

(iii) One then inserts the expression for the canonical variables in terms of the prepotentials inside the action and obtains (4.13).

\section{B. Solving the momentum constraint}

We first solve the momentum constraint. This constraint reads [45]

$$
\mathcal{C}^{i_{1} \cdots i_{s-1}} \equiv \partial_{k} \pi^{k i_{1} \cdots i_{s-1}}+“ \text { more } "=0
$$

where "more" stands for terms that are linear in the second order derivatives of $\alpha_{i_{1} \cdots i_{s-3}}$, which one can set to zero by a suitable gauge transformation. In the gauges where "more" vanishes, the constraint reduces to

$$
\partial_{k} \pi^{k i_{1} \cdots i_{s-1}}=0,
$$

the general solution of which is given by $\pi^{i_{1} \cdots i_{s}}=G^{i_{1} \cdots i_{s}}[P]$ $[27,28]$. Here $G^{i_{1} \cdots i_{s}}[P]$ is the Einstein tensor of some prepotential $P_{i_{1} \cdots i_{s}}$ which is totally symmetric.

For fixed momentum $\pi^{i_{1} \cdots i_{s}}$, the prepotential $P_{i_{1} \cdots i_{s}}$ is determined up to a spin- $s$ diffeomorphism. However, there is a residual gauge freedom in the above gauges, so that $\pi^{i_{1} \cdots i_{s}}$ is not completely fixed. It is straightforward but somewhat tedious to check that the residual gauge symmetry is accounted for by a spin- $s$ Weyl transformation of the prepotential $P_{i_{1} \ldots i_{s}}$, which therefore enjoys all the gauge symmetries of a conformal spin-s field.

These results extend what was found earlier for spins 2 [23] and 3 [21]. It is instructive to exhibit explicitly the formulas in the case of spin 4, which illustrates all the points and still yields readable expressions.

The momentum constraint reads in this case

$$
\begin{aligned}
0= & 4 \partial^{n} \pi_{\mathrm{klmn}}+6 \delta_{(k l} \Delta \alpha_{m)} \\
& -10 \delta_{(k l} \partial_{m)} \partial^{n} \alpha_{n}
\end{aligned}
$$

and the gauge freedom is

$$
\begin{aligned}
\delta \pi_{\mathrm{klmn}}= & -12 \partial_{(k} \partial_{l} \Xi_{m n)} \\
& +12 \delta_{(k l}\left(\Delta \Xi_{m n)}+\partial_{m} \partial^{p} \Xi_{n) p}\right) \\
& +4 \delta_{(k l} \delta_{m n)}\left(2 \Delta \bar{\Xi}+3 \partial^{p} \partial^{q} \Xi_{p q}\right), \\
& \delta \alpha_{k}=-6 \partial^{l} \Xi_{k l}-2 \partial_{k} \bar{\Xi} .
\end{aligned}
$$

The residual gauge transformations in the gauge $3 \Delta \alpha_{k}-5 \partial_{k} \partial^{l} \alpha_{l}=0$, which eliminates the $\alpha$-terms from the constraint, must fulfill

$$
0=-18 \partial^{l} \Delta \Xi_{k l}+4 \partial_{k} \Delta \bar{\Xi}+30 \partial_{k} \partial^{l} \partial^{m} \Xi_{l m} .
$$

The divergence of this equation gives (after acting with $\left.\Delta^{-1}\right), 3 \partial^{k} \partial^{l} \Xi_{k l}+\Delta \bar{\Xi}=0$. Substituting this finding in the previous equation yields, after acting again with $\Delta^{-1}$, $3 \partial^{l}\left(\Xi_{k l}+\frac{1}{3} \delta_{k l} \bar{\Xi}\right)=0$. This is the divergence of a symmetric tensor, so the solution is the double divergence of a tensor with the symmetry of the Riemann tensor:

$$
\Xi_{k l}+\frac{1}{3} \delta_{k l} \bar{\Xi}=\partial^{m} \partial^{n} \Theta_{\mathrm{mknl}} \cdot
$$

Therefore, one has

$$
\Xi_{k l}=\partial^{m} \partial^{n} \Theta_{\mathrm{mknl}}-\frac{1}{6} \delta_{k l} \partial^{m} \partial_{n} \Theta_{m p}{ }^{n p}
$$

This class of gauge transformations can be checked to give a zero variation not only to the contribution of $\alpha_{k}$ to the constraint but in fact also to $\alpha_{k}$ itself.

We can dualize $\Theta_{\mathrm{klmn}}=\epsilon_{k l p} \epsilon_{m n q} \theta^{p q}$, with a symmetric $\theta_{k l}$, to obtain:

$$
\begin{aligned}
\Xi_{k l}= & \frac{5}{6} \delta_{k l}\left(\Delta \bar{\theta}-\partial^{m} \partial^{n} \theta_{m n}\right) \\
& +2 \partial_{(k} \partial^{m} \theta_{l) m}-\partial_{k} \partial_{l} \bar{\theta}-\Delta \theta_{k l} .
\end{aligned}
$$

The gauge transformation of $\pi_{\mathrm{klmn}}$ with this parameter is found then to be exactly the Einstein tensor of a Weyl diffeomorphism

$$
\delta \pi_{\mathrm{klmn}}=G_{\mathrm{klmn}}\left[12 \delta_{(p q} \theta_{r s)}\right] .
$$

Once the spin-4 momentum constraint has been brought in the standard form $\partial^{k} \pi_{\mathrm{klmn}}=0$ by partial gauge fixing, it can be solved by the familiar techniques recalled at the beginning of this section for general $s$. As it is known $[27,28]$, the general solution of the equation $\partial^{k} \pi_{\mathrm{klmn}}=0$ is indeed the Einstein tensor of a symmetric tensor $P_{\text {klmn }}$, which is the prepotential for the momenta:

$$
\pi_{\mathrm{klmn}}=G_{\mathrm{klmn}}[P] .
$$


Since the gauge freedom of $\pi_{\mathrm{klmn}}$ is given by the Einstein tensor of a Weyl transformation, the gauge freedom of the prepotential $P_{\text {klmn }}$ will be given by a spin-4 Weyl transformation and a spin-4 diffeomorphism:

$$
\delta P_{\mathrm{klmn}}=4 \partial_{(k} \xi_{l m n)}+6 \delta_{(k l} \lambda_{m n)},
$$

the first term corresponding to all the transformations of the prepotential leaving $\pi_{\mathrm{klmn}}$ invariant and the second to those realizing on it a residual gauge transformation.

The solution in a general gauge will be given by these expressions to which are added general gauge transformation terms, parametrized by further prepotentials that drop from the action by gauge invariance and which are usually not considered for that reason.

\section{Solving the Hamiltonian constraint}

We now solve the Hamiltonian constraint. The functions $\mathcal{C}_{i_{1} \cdots i_{s-2}}$ are linear in the second order derivatives of the spin$s$ field $h_{i_{1} \cdots i_{s}}$ and linear in the first order derivatives of the conjugate momenta $\tilde{\Pi}^{i_{1} \cdots i_{s-3}}$. One may rewrite these constraints in the equivalent form (suppressing indices)

$$
\Psi-d_{(s-2)} \tilde{\Pi}=0
$$

where $\Psi$ is the function of the second order derivatives of the spin-s field with Young symmetry

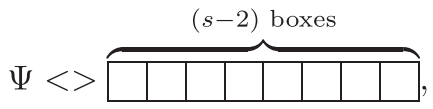

introduced in [21], such that ${ }^{*} \bar{G} \equiv{ }^{*} G^{[1]}=d_{(s-2)}^{s-2} \Psi$, where * denotes here the dual on all indices.

The results of $[27,28]$ applied to $d_{(s-2)}$ such that $d_{(s-2)}^{s-1}=0$ imply that Eq. (5.14) is completely equivalent to $d_{(s-2)}^{s-2} \Psi=0$, i.e., $G^{[1]}[h]=0$. As we have recalled, this equation implies in turn the existence of a prepotential $Z$ for $h$ (continuing to omit indices) such that the Einstein tensor of $h$ is equal to the Cotton tensor of $Z$. Once $h$ is expressed in terms of $Z$, the expression $d_{(s-2)}^{s-2} \Psi[Z]$ identically vanishes, implying according to the generalized algebraic Poincaré lemma of $[27,28]$ that one can write $\Psi[Z]=$ $d_{(s-2)} \tilde{\Pi}[Z]$, for some $\tilde{\Pi}[Z]$.

This completely solves the Hamiltonian constraint in terms of the prepotential $Z_{i_{1} \cdots i_{2}}$. By construction, this prepotential has the gauge symmetry of a conformal spin-s field.

The procedure is direct for spins 1 , where there is no Hamiltonian constraint, and 2, where one gets directly $G^{[1]}[h]=0$ without having to differentiate. It was detailed for spin 3 in [21]. It is again instructive to illustrate it explicitly for the spin 4 field, where the formulas remain readable.
The Hamiltonian constraint for the spin 4 is

$$
\begin{aligned}
\mathcal{C}_{k l} \equiv & 3 \partial_{(k} \tilde{\Pi}_{l)}+\delta_{k l} \partial^{m} \tilde{\Pi}_{m} \\
& -6\left(\Delta \bar{h}_{k l}-\partial^{m} \partial^{n} h_{\mathrm{klmn}}+\partial_{(k} \partial^{m} \bar{h}_{l) m}+\partial_{k} \partial_{l} \overline{\bar{h}}\right) \\
& -4 \delta_{k l} \Delta \overline{\bar{h}}=0,
\end{aligned}
$$

The gauge freedom of $h_{\mathrm{klmn}}$ and $\tilde{\Pi}_{k}$ is

$$
\begin{gathered}
\delta \tilde{\Pi}_{k}=6 \Delta \bar{\xi}_{k}+10 \partial_{k} \partial^{l} \bar{\xi}_{l}, \\
\delta h_{\mathrm{klmn}}=4 \partial_{(k} \xi_{l m n)} .
\end{gathered}
$$

One can equivalently rewrite the constraint as

$$
\mathcal{C}_{k l}-\frac{1}{6} \delta_{k l} \overline{\mathcal{C}} \equiv 3 \partial_{(k} \tilde{\Pi}_{l)}-6 \Psi_{k l}=0,
$$

where $\overline{\mathcal{C}}$ is the trace of $\mathcal{C}_{k l}$ and

$$
\begin{aligned}
\Psi_{k l} \equiv & \Delta \bar{h}_{k l}-\partial^{m} \partial^{n} h_{\mathrm{klmn}} \\
& +\partial_{(k} \partial^{m} \bar{h}_{l) m}+\partial_{k} \partial_{l} \overline{\bar{h}}
\end{aligned}
$$

One has

$$
\epsilon_{k m p} \epsilon_{l n q} \partial^{m} \partial^{n} \Psi^{p q}=\bar{G}_{k l}[h]
$$

where $\bar{G}_{k l}$ is the trace of the Einstein tensor $G_{\mathrm{klmn}}[h]$ of $h_{\mathrm{klmn}}$. So, one gets:

$$
\bar{G}_{k l}[h]=-\frac{1}{6} \epsilon_{k m p} \epsilon_{l n q} \partial^{m} \partial^{n} \tilde{\mathcal{C}}^{p q}
$$

with $\tilde{\mathcal{C}}^{p q} \equiv \mathcal{C}^{p q}-\frac{1}{6} \delta^{p q} \overline{\mathcal{C}}$.

The Hamiltonian constraint implies $\bar{G}_{k l}[h]=0$. Therefore, the theorem of [21] yields $G_{i j k l}[h]=B_{i j k l}[Z]$ for some prepotential $Z_{\mathrm{klmn}}$, where $B_{i j k l}$ is the Cotton tensor. Explicitly,

$$
\begin{aligned}
h_{i j k l}[Z]= & \epsilon_{(i \mid m n} \partial^{m}\left[-\Delta Z^{n}{ }_{\mid j k l)}+\frac{1}{2} \delta_{\mid j k} \Delta \bar{Z}^{n}{ }_{l)}\right. \\
& \left.-\frac{1}{2} \delta_{\mid j k} \partial^{p} \partial^{q} Z^{n}{ }_{l) p q}\right]+4 \partial_{(i} \omega_{j k l)},
\end{aligned}
$$

where we have added a spin- 4 diffeomorphism term parametrized by $\omega_{j k l}$.

Direct substitution gives then

$$
\begin{aligned}
\Psi_{i j}= & \partial_{(i \mid}\left[3 \Delta \bar{\omega}_{j)}+5 \partial_{\mid j)} \partial^{k} \bar{\omega}_{k}\right. \\
& \left.-\frac{1}{8} \epsilon_{\mid j) m n} \partial^{m}\left(\partial^{k} \Delta \bar{Z}^{n}{ }_{k}+\partial^{p} \partial^{q} \partial^{k} Z^{n}{ }_{k p q}\right)\right]
\end{aligned}
$$

from which one derives, using the constraint, the following expression for $\tilde{\Pi}_{i}$, 


$$
\begin{aligned}
\tilde{\Pi}_{i}= & 6 \Delta \bar{\omega}_{i}+10 \partial_{i} \partial^{k} \bar{\omega}_{k} \\
& -\frac{1}{4} \epsilon_{i m n} \partial^{m}\left(\partial^{k} \Delta \bar{Z}^{n}{ }_{k}+\partial^{p} \partial^{q} \partial^{k} Z_{k p q}^{n}\right) .
\end{aligned}
$$

One could in fact add a solution $\kappa_{i}$ of the equation $\partial_{(i} \kappa_{j)}=0$ (Killing equation) to $\tilde{\Pi}_{i}$ but we do not consider this possibility here by assuming for instance appropriate boundary conditions (vanishing of all fields at infinity eliminates $\kappa_{i} \sim C_{i}+\mu_{i j} x^{j}$, where $C_{i}$ and $\mu_{i j}=-\mu_{j i}$ are constants).

\section{Hamiltonian action in terms of prepotentials}

The elimination of the canonical variables in terms of the prepotentials in the action is a rather burdensome task. However, the derivation can be considerably short-cut by invariance arguments.

The kinetic term in the action is quadratic in the prepotentials $Z$ and $P$ and involves $2 s-1$ spatial derivatives, and one time derivative. Furthermore, it must be invariant under spin- $s$ diffeomorphisms and spin-s Weyl transformations of both prepotentials. This implies, making integrations by parts if necessary, that the kinetic term has necessarily the form of the kinetic term of the action (4.13) upon identification of the prepotential $Z$ with the prepotential $Z^{1}$ and the prepotential $P$ with the prepotential $Z^{2}$.

Similarly, the Hamiltonian is the integral of a quadratic expression in the prepotentials $Z$ and $P$ involving $2 s$ spatial derivatives. By spin- $s$ diffeomorphism invariance, it can be written as the integral of a quadratic expression in their Einstein tensors and their successive traces-or equivalently, the Schouten tensors and their successive traces. As we have seen, Weyl invariance fixes then the coefficients up to an overall rescaling, so that the Hamiltonian takes necessarily the form (4.14), but with $\delta_{a b}$ that might be replaced by a diagonal $\mu_{a b}$ with eigenvalues different from 1. However, given that the equations of motion following from the action (4.13) are, as we have seen, consequences of the Fronsdal equations, they cannot be in contradiction with the equations of motion following from the Hamiltonian action (equivalent to the Fronsdal equations) and this is possible only if $\mu_{a b}=\delta_{a b}$.

One thus concludes that the action (4.13) for twisted selfduality is indeed the Hamiltonian form of the Fronsdal action with the constraints solved for in terms of prepotentials. This shows in particular that the electric fieldmagnetic field version of twisted self-duality obtained by considering only the spatial components of (2.11) form indeed a complete set, as announced. The analysis also shows that duality-conjugate and canonically conjugate are equivalent (up to field-independent factors).

\section{ADDITIONAL CONSIDERATIONS}

\section{A. Higher dimensions and twisted self-duality}

In higher spacetime dimension $D$, the equations of motion can also be reformulated as twisted self-duality conditions on the curvatures of the spin-s field and its dual. What is new is that the dual of a spin-s field is not given by a symmetric tensor, but by a tensor of mixed Young symmetry type

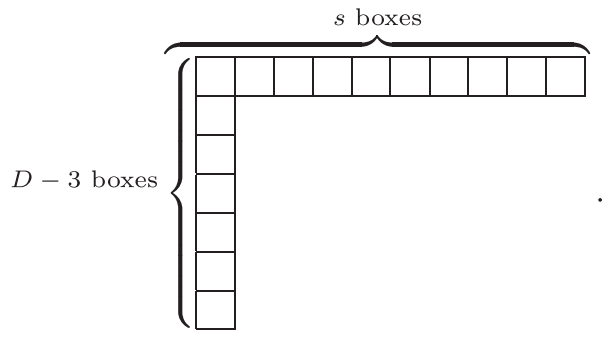

Consequently, the curvature tensor and its dual are also tensors of different types. Nevertheless, the electric (respectively, magnetic) field of the spin- $s$ field is a spatial tensor of the same type as the magnetic (respectively, electric) field of its dual and the twisted self-duality conditions again equate them [up to \pm similarly to Eq. (3.11)]. The electric and magnetic fields are subject to tracelessness constraints that can be solved in terms of appropriate prepotentials, which are the variables for the variational principle from which the twisted self-duality conditions derive. Again, this variational principle is equivalent to the Hamiltonian variational principle.

We have not worked out the specific derivation for all spins in higher dimensions $D$, but the results of [17] for the spin-2 case, together with our above analysis, make us confident that this derivation indeed goes through as described here.

\section{B. Manifest $S O(2)$ electric-magnetic duality invariance in $D=4$}

In $D=4$ spacetime dimensions, the spin-s field and its dual are tensors of the same type, as we have seen. The equations enjoy then $S O(2)$ electric-magnetic duality invariance that rotates the field and its dual in the internal two-dimensional space that they span. This comes over and above the twisted self-duality reformulation.

The $S O(2)$ electric-magnetic duality invariance amounts to perform rotations in the internal space of the prepotentials. It is clear that it is also a symmetry of the action (4.13). Thus, the prepotential reformulation makes it obvious that $S O(2)$ electric-magnetic duality invariance is a manifest off-shell symmetry, and not just a symmetry of the equations of motion.

\section{COMMENTS AND CONCLUSIONS}

In this paper, we have achieved two things. (i) First, we have rewritten the equations of motion for higher spin gauge fields as twisted self-duality conditions, in which the spin- $s$ field and its dual are put on exactly the same footing. (ii) Second, by introducing prepotentials for the spin-s field and its dual, we have shown how these equations derive 
from a variational principle, providing thereby a duality symmetric formulation of the theory.

One observes again, for all spins, the intriguing emergence of higher spin Weyl gauge symmetries [46-50] for the prepotentials, in addition to spin- $s$ diffeomorphisms. This generalizes what was found in the spin-2 case in [23].

One should also stress the remarkable simplicity of the final action. Furthermore, this final action takes the same form for all spins. This uniformity suggests that the prepotential formalism might perhaps be a good starting point for exploring the symmetries mixing all spins-in particular the $s p(8)$-symmetry in four spacetime dimensions [51,52].

We have restricted the analysis to massless fields in flat spacetime. Extension to constant curvature backgrounds [24] and to partially massless fields would be of definite interest [53-55].

\section{ACKNOWLEDGMENTS}

We thank Andrea Campoleoni for useful discussions. A. L. is Research Fellow at the Belgian F. R. S.-FNRS. The work of S. H. has been supported by the Fondecyt Grant No. 3160781. This work was partially supported by the ERC Advanced Grants "SyDuGraM" and "High-SpinGrav", by F. R. S.-FNRS through the conventions PDRT.1025.14 and IISN-4.4503.15, and by the "Communauté Française de Belgique" through the ARC program. The Centro de Estudios Científicos (CECs) is funded by the Chilean Government through the Centers of Excellence Base Financing Program of Conicyt.

\section{APPENDIX A: PREPOTENTIALS FOR EVEN SPINS}

We give in this appendix the form of the spin-s field $h_{i_{1} \cdots i_{s}}$ in terms of the corresponding prepotential $Z_{i_{1} \cdots i_{s}}$ when $s$ is even. The case of an odd $s$ is treated in Appendix B.

Because of the gauge symmetries, the expression $h[Z]$ is not unique. To any solution, one may add a gauge transformation term. Our particular solution corresponds to a definite choice.

Our strategy is as follows: (i) First, one writes the most general form for $h$ in terms of $Z$ compatible with the index structure and the fact that it contains $s-1$ derivatives. (ii) Second, one fixes the coefficients of the various terms such that a gauge transformation of $Z$ induces a gauge transformation of $h$. This turns out to completely fix $h[Z]$ up to an overall multiplicative constant. (iii) Third, one fixes that multiplicative constant through the condition $G[h[Z]]=D[Z]$, which we impose and verify in a convenient gauge for $Z$.

\section{First step}

A generic term in the expression for $h_{i_{1} \cdots i_{s}}$ in terms of $Z_{i_{1} \cdots i_{s}}$ involves one Levi-Civita tensor when $s$ is even, as well as $s-1$ derivatives of $Z$. It can also contain a product of $p \delta_{i_{i} i_{k}}$ 's with free indices among $i_{1}, i_{2}, \ldots, i_{s}$. Hence a generic term takes the form

$$
\delta_{i_{1} i_{2}} \cdots \delta_{i_{2 p-1} i_{2 p}} \epsilon_{k_{1} k_{2} k_{3}} \partial_{m_{1}} \cdots \partial_{m_{s-1}} Z_{j_{1} \cdots j_{s}}
$$

for some $p$ such that $0 \leq p \leq n-1$ where $s=2 n$ ( $p$ cannot be equal to $n$ since the Levi-Civita symbol must necessarily carry a free index, see below, so that there must be at least one free index left). Among the indices $k_{1}, k_{2}, k_{3}, m_{1}, \ldots, m_{s-1}, j_{1}, j_{2}, \ldots, j_{s}$, there are $s-2 p$ indices equal to the remaining $i_{a}$ 's, and the other indices are contracted with $\delta^{a b}$ 's. There is also an implicit symmetrization over the free indices $i_{a}$, taken as before to be of weight one.

The structure of the indices of the Levi-Civita symbol is very clear: because of the symmetries, one index is a free index $i_{b}$, one index is contracted with a derivative operator, and one index is contracted with an index of $Z$. Furthermore, if an index $m_{b}$ on the derivatives is equal to one of the free indices $i_{b}$, then, the term can be removed by a gauge transformation. This means that apart from one index contracted with an index of the Levi-Civita tensor, the remaining indices on the derivative operators are necessarily contracted either among themselves to produce Laplacians or with indices of $Z$. In other words, the remaining free indices, in number $s-2 p-1$ are carried by $Z$. One index on $Z$ is contracted with one index of the Levi-Civita tensor as we have seen, and the other indices on $Z$, in number $2 p$, are contracted either among themselves to produce traces or with the indices carried by the derivative operators. Thus, if we know the number of traces that occur in $Z$, say $q$, the structure of the term (A1) is completely determined,

$$
\begin{aligned}
& \delta_{i_{1} i_{2}} \cdots \delta_{i_{2 p-1} i_{2 p}} \epsilon_{i_{2 p+1} k}^{t} \partial^{k} \partial^{j_{1}} \cdots \partial^{j_{2 p-2 q}} \Delta^{n-1-p+q} \\
& Z_{i_{2 p+2} \cdots i_{s} t j_{1} \cdots j_{2 p-2 q}}^{[q]}
\end{aligned}
$$

or, in symbolic form,

$$
\delta^{p}(\epsilon \cdot \partial \cdot)(\partial \cdot \partial \cdot)^{p-q} \Delta^{n-1-p+q} Z^{[q]} .
$$

One has $0 \leq q \leq p$ and complete symmetrization on the free indices $i_{b}$ is understood.

Accordingly, the expression for $h_{i_{1} \cdots i_{s}}$ in terms of $Z_{i_{1} \cdots i_{s}}$ reads

$h=\sum_{p=0}^{n-1} \sum_{q=0}^{p} a_{p, q} \delta^{p}(\epsilon \cdot \partial \cdot)(\partial \cdot \partial \cdot)^{p-q} \Delta^{n-1-p+q} Z^{[q]}$.

where the coefficients $a_{p, q}$ are determined next. 


\section{Second step}

By requesting that a gauge transformation of $Z_{i_{1} i_{2} \cdots i_{s}}$ induces a gauge transformation of $h_{i_{1} \cdots i_{s}}$, the coefficients $a_{p, q}$ are found to be given by

$$
\begin{aligned}
a_{p, q}= & 2^{-p}(-)^{q} \\
& \times \frac{(n-1) !(2 n-p-1) !(2 n-1) ! !}{q !(p-q) !(n-p-1) !(2 n-1) !(2 n-2 p-1) ! !} a
\end{aligned}
$$

where the multiplicative constant $a$ is undetermined at this stage. The double factorial of an odd number $2 k+1$ is equal to the product of all the odd numbers up to $2 k+1$,

$$
(2 k+1) ! !=1 \cdot 3 \cdot 5 \cdots(2 k-1)(2 k+1)
$$

The computation is fastidious but conceptually straightforward and left to the reader.

\section{Third step}

Finally, we fix the remaining coefficient $a$ by imposing that $G[h[Z]]=D[Z]$. This is most conveniently done in the gauge

$$
\partial^{i_{1}} Z_{i_{1} \cdots i_{s}}=0, \quad Z^{i_{1}}{ }_{i_{1} i_{3} \cdots i_{s}}=0
$$

(transverse, traceless gauge). This gauge is permissible given that the gauge transformations of the prepotential involves both spin- $s$ diffeomorphisms and spin-s Weyl transformations. In that gauge, the Cotton tensor reduces to

$$
D[Z]_{i_{1} i_{2} \cdots i_{s}}=-\epsilon_{\left(i_{1} \mid j k\right.} \partial^{j} \Delta^{2 n-1} Z^{k}{ }_{\left.\mid i_{2} \cdots i_{s}\right)}
$$

or in symbolic form,

$$
D[Z]=-(\epsilon \cdot \partial \cdot) \Delta^{2 n-1} Z,
$$

while $h_{i_{1} \cdots i_{s}}$ is also divergenceless and traceless (which shows, incidentally, that on the $\bar{G}[h]=0$ shell, one may impose both conditions also on $h$ ) and its Einstein tensor, expressed in terms of $Z$, becomes

$$
\begin{aligned}
G[h[Z]]_{i_{1} \cdots i_{s}} & =(-)^{n} \Delta^{n} h[Z]_{i_{1} \cdots i_{s}} \\
& =(-)^{n} a \epsilon_{\left(i_{1} \mid j k\right.} \partial^{j} \Delta^{2 n-1} Z^{k}{ }_{\left.\mid i_{2} \cdots i_{s}\right)}
\end{aligned}
$$

i.e.,

$$
G[h[Z]]=(-)^{n} a \epsilon \cdot \partial \cdot \Delta^{2 n-1} Z .
$$

This shows that

$$
a=-(-)^{n}
$$

and completes the determination of $h$ in terms of its prepotential $Z$.

\section{APPENDIX B: PREPOTENTIALS FOR ODD SPINS}

The procedure for odd spins follows the same steps, but now there is no Levi-Civita tensor involved in the expression $h[Z]$ since there is an even number of derivatives.

\section{First step}

A generic term in the expression for $h_{i_{1} \cdots i_{s}}$ in terms of $Z_{i_{1} \cdots i_{s}}$ involves $s-1$ derivatives of $Z$. It can also contain a product of $p \delta_{i_{j} i_{k}}$ 's with free indices among $i_{1}, i_{2}, \ldots, i_{s}$. Hence a generic term takes the form

$$
\delta_{i_{1} i_{2}} \cdots \delta_{i_{2 p-1} i_{2 p}} \partial_{m_{1}} \cdots \partial_{m_{s-1}} Z_{j_{1} \cdots j_{s}}
$$

for some $p$ such that $0 \leq p \leq n$ where $s=2 n+1$. Among the indices $m_{1}, \ldots, m_{s-1}, j_{1}, j_{2}, \ldots, j_{s}$, there are $s-2 p$ indices equal to the remaining $i_{a}$ 's, and the other indices are contracted with $\delta^{a b}$ 's. There is also an implicit symmetrization over the free indices $i_{a}$.

Again, if an index $m_{b}$ on the derivatives is equal to one of the free indices $i_{b}$, then, the term can be removed by a gauge transformation. This means that the indices on the derivative operators are necessarily contracted either among themselves to produce Laplacians or with indices of $Z$. In other words, the remaining free indices, in number $s-2 p$ are carried by $Z$. The other indices on $Z$, in number $2 p$, are contracted either among themselves to produce traces or with the indices carried by the derivative operators. Thus, if we know the number of traces that occur in $Z$, say $q$, the structure of the term (B1) is completely determined, as in the even spin case

$$
\begin{aligned}
\delta_{i_{1} i_{2}} \cdots \delta_{i_{2 p-1} i_{2 p}} \partial^{j_{1}} \cdots & \partial^{j_{2 p-2 q}} \Delta^{n-p+q} \\
& Z_{i_{2 p+1} \cdots i_{s} j_{1} \cdots j_{2 p-2 q}}^{[q]},
\end{aligned}
$$

or, in a more compact way:

$$
\delta^{p}(\partial \cdot \partial \cdot)^{p-q} \Delta^{n-p+q} Z^{[q]} .
$$

One has $0 \leq q \leq p$ and complete symmetrization on the free indices $i_{b}$ is understood.

Accordingly, the expression for $h_{i_{1} \cdots i_{s}}$ in terms of $Z_{i_{1} \cdots i_{s}}$ reads

$$
h=\sum_{p=0}^{n} \sum_{q=0}^{p} a_{p, q} \delta^{p}(\partial \cdot \partial \cdot)^{p-q} \Delta^{n-p+q} Z^{[q]} .
$$

where the coefficients $a_{p, q}$ are determined in the second step.

\section{Second step}

By requesting that a gauge transformation of $Z_{i_{1} i_{2} \cdots i_{s}}$ induces a gauge transformation of $h_{i_{1} \cdots i_{s}}$, the coefficients 
$a_{p, q}$ are found to be given up to an overall multiplicative constant $a$ by

$a_{p, q}=(-)^{q} 2^{-p} \frac{n !(2 n-p) !(2 n+1) ! !}{q !(p-q) !(n-p) !(2 n) !(2 n-2 p+1) ! !} a$.

The computation is again somewhat fastidious but conceptually straightforward and left to the reader.

\section{Third step}

Finally, we fix the remaining coefficient $a$ by imposing that $G[h[Z]]=D[Z]$. This is most conveniently done in the transverse, traceless gauge for $Z$

$$
\partial^{i_{1}} Z_{i_{1} \cdots i_{s}}=0, \quad Z^{i_{1}}{ }_{i_{1} i_{3} \cdots i_{s}}=0
$$

which is again permissible. In that gauge, the Cotton tensor reduces to

$$
D[Z]=(\epsilon \cdot \partial \cdot) \Delta^{2 n} Z
$$

while the Einstein tensor of $h[Z]$ becomes

$$
G[h[Z]]=(-)^{n} a \epsilon \cdot \partial \cdot \Delta^{2 n} Z .
$$

This leads to

$$
a=(-)^{n}
$$

and completes the determination of $h$ in terms of its prepotential $Z$.
[1] J. Ehlers, Ph.D., Hamburg University (1957); Les Theories relativistes de la gravitation, Colloques Internationaux du CNRS 91, 275 (1962).

[2] E. Cremmer and B. Julia, The $\mathrm{SO}(8)$ supergravity, Nucl. Phys. B159, 141 (1979).

[3] S. Ananth, L. Brink, and S. Majumdar, Exceptional versus superPoincaré algebra as the defining symmetry of maximal supergravity, J. High Energy Phys. 03 (2016) 051.

[4] B. Julia, in Superspace and Supergravity, edited by S. W. Hawking and M. Roćek, Nuffield Gravity Workshop, Cambridge, England, June 22-July 12, 1980 (Cambridge University Press, Cambridge, England, 1981).

[5] B. Julia, in Proc. Johns Hopkins Workshop on "Current Problems in Particle Physics, Unified Theories and Beyond" (Johns Hopkins University, Baltimore, 1984), p. 23, preprint LPTENS-81-14.

[6] B. Julia, Kac-Moody Symmetry of Gravitation and Supergravity, Lectures in Applied Mathematics (American Mathematical Society, Providence, RI, 1985), Vol. 21, p. 355, preprint LPTENS-82-22.

[7] H. Nicolai, A hyperbolic Lie algebra from supergravity, Phys. Lett. B 276, 333 (1992).

[8] B. L. Julia, Dualities in the classical supergravity limits: Dualizations, dualities and a detour via $(4 k+2)$ dimensions, arXiv:hep-th/9805083.

[9] P. C. West, E(11) and M theory, Classical Quantum Gravity 18, 4443 (2001).

[10] T. Damour, M. Henneaux, and H. Nicolai, E(10) and a Small Tension Expansion, of M Theory, Phys. Rev. Lett. 89, 221601 (2002).

[11] E. Cremmer, B. Julia, H. Lu, and C. N. Pope, Dualization of dualities. 1., Nucl. Phys. B523, 73 (1998).

[12] E. Cremmer, B. Julia, H. Lu, and C. N. Pope, Dualisation of dualities. II: Twisted self-duality of doubled fields and superdualities, Nucl. Phys. B535, 242 (1998).
[13] A. G. Tumanov and P. West, E11 must be a symmetry of strings and branes, arXiv:1512.01644; , E11 in 11D, Phys. Lett. B 758, 278 (2016).

[14] C. Bunster and M. Henneaux, The action for twisted selfduality, Phys. Rev. D 83, 125015 (2011).

[15] C. Bunster and M. Henneaux, Duality Invariance Implies Poincaré Invariance, Phys. Rev. Lett. 110, 011603 (2013).

[16] C. Bunster, M. Henneaux, and S. Hörtner, Gravitational electric-magnetic duality, gauge invariance and twisted self-duality, J. Phys. A 46, 214016 (2013); Corrigendum, J. Phys. A 46, 269501 (2013).

[17] C. Bunster, M. Henneaux, and S. Hörtner, Twisted selfduality for linearized gravity in D dimensions, Phys. Rev. D 88, 064032 (2013).

[18] D. J. Gross, High-Energy Symmetries of String Theory, Phys. Rev. Lett. 60, 1229 (1988).

[19] It should be stressed that it is not clear whether dynamical higher spin gauge fields do actually arise in the simplest nonlinear realizations of the $E_{10}$ or other conjectured hidden symmetries of a similar type, see M. Henneaux, A. Kleinschmidt, and H. Nicolai, Real forms of extended Kac-Moody symmetries and higher spin gauge theories, Gen. Relativ. Gravit. 44, 1787 (2012). However, it is not unreasonable to expect that the ultimate theory will combine both one of these hidden algebras and a Vasiliev higher spin algebra. In any case, twisted self-duality for higher spin gauge fields is interesting in itself as it reveals interesting properties of the higher spin gauge field dynamics.

[20] X. Bekaert and N. Boulanger, On geometric equations and duality for free higher spins, Phys. Lett. B 561, 183 (2003).

[21] M. Henneaux, S. Hörtner, and A. Leonard, Higher spin conformal geometry in three dimensions and prepotentials for higher spin gauge fields, J. High Energy Phys. 01 (2016) 073. 
[22] S. Deser and C. Teitelboim, Duality transformations of Abelian and non-Abelian gauge fields, Phys. Rev. D 13, 1592 (1976).

[23] M. Henneaux and C. Teitelboim, Duality in linearized gravity, Phys. Rev. D 71, 024018 (2005).

[24] B. Julia, J. Levie, and S. Ray, Gravitational duality near de Sitter space, J. High Energy Phys. 11 (2005) 025.

[25] For a different approach to $S O(2)$ electric-magnetic duality invariance of hifger spins, see: S. Deser and D. Seminara, Duality invariance of all free bosonic and fermionic gauge fields, Phys. Lett. B 607, 317 (2005).

[26] B. de Wit and D. Z. Freedman, Systematics of higher spin gauge fields, Phys. Rev. D 21, 358 (1980).

[27] M. Dubois-Violette and M. Henneaux, Generalized cohomology for irreducible tensor fields of mixed Young symmetry type, Lett. Math. Phys. 49, 245 (1999).

[28] M. Dubois-Violette and M. Henneaux, Tensor fields of mixed Young symmetry type and $\mathrm{N}$ complexes, Commun. Math. Phys. 226, 393 (2002).

[29] P. J. Olver, University of Minnesota, Mathematics Report No. 82-101, 1982.

[30] X. Bekaert and N. Boulanger, Tensor gauge fields in arbitrary representations of GL(D,R): Duality and Poincaré lemma, Commun. Math. Phys. 245, 27 (2004).

[31] An alternative way, which leads to a reducible theory, imposes transverse conditions instead of tracelessness conditions: A. Campoleoni and D. Francia, Maxwell-like Lagrangians for higher spins, J. High Energy Phys. 03 (2013) 168.

[32] C. Fronsdal, Massless fields with integer spin, Phys. Rev. D 18, 3624 (1978).

[33] D. Francia and A. Sagnotti, Free geometric equations for higher spins, Phys. Lett. B 543, 303 (2002).

[34] C. M. Hull, Duality in gravity and higher spin gauge fields, J. High Energy Phys. 09 (2001) 027.

[35] T. Damour and S. Deser, Geometry of spin 3 gauge theories, Annales Poincare Phys. Theor. 470, 277 (1987).

[36] C. N. Pope and P. K. Townsend, Conformal higher spin in $(2+1)$-dimensions, Phys. Lett. B 225, 245 (1989).

[37] E. A. Bergshoeff, O. Hohm, and P. K. Townsend, On higher derivatives in $3 \mathrm{D}$ gravity and higher spin gauge theories, Ann. Phys. (Amsterdam) 325, 1118 (2010).

[38] E. A. Bergshoeff, M. Kovacevic, J. Rosseel, P. K. Townsend, and Y. Yin, A spin-4 analog of 3D massive gravity, Classical Quantum Gravity 28, 245007 (2011).
[39] B.E. W. Nilsson, Towards an exact frame formulation of conformal higher spins in three dimensions, J. High Energy Phys. 09 (2015) 078.

[40] B. E. W. Nilsson, On the conformal higher spin unfolding equation for a three-dimensional self-interacting scalar field, J. High Energy Phys. 08 (2016) 142.

[41] H. Linander and B. E. W. Nilsson, The non-linear coupled spin 2-spin 3 Cotton equation in three dimensions, J. High Energy Phys. 07 (2016) 024.

[42] S. M. Kuzenko and D. X. Ogburn, Off-shell higher spin supermultiplets in three dimensions, High Energy Phys. arXiv:1603.04668 [(to be published)].

[43] S. M. Kuzenko, Higher-spin extensions of the linear-chiral duality in three dimensions, arXiv:1606.08624.

[44] R. R. Metsaev, Extended Hamiltonian action for arbitrary spin fields in flat and AdS spaces, J. Phys. A 46, 214021 (2013).

[45] A. Campoleoni, M. Henneaux, S. Hörtner, and A. Leonard, Higher-spin charges in Hamiltonian form. I. Bose fields, J. High Energy Phys. 10 (2016) 146.

[46] E. S. Fradkin and A. A. Tseytlin, Conformal supergravity, Phys. Rep. 119, 233 (1985).

[47] M. A. Vasiliev, Conformal higher spin symmetries of 4-d massless supermultiplets and $\mathrm{osp}(\mathrm{L}, 2 \mathrm{M})$ invariant equations in generalized (super)space, Phys. Rev. D 66, 066006 (2002).

[48] A. Y. Segal, Conformal higher spin theory, Nucl. Phys. B664, 59 (2003).

[49] O. V. Shaynkman, I. Y. Tipunin, and M. A. Vasiliev, Unfolded form of conformal equations in $\mathrm{M}$ dimensions and o $(\mathrm{M}+2)$ modules, Rev. Math. Phys. 18, 823 (2006).

[50] R. R. Metsaev, Ordinary-derivative formulation of conformal totally symmetric arbitrary spin bosonic fields, J. High Energy Phys. 06 (2012) 062.

[51] C. Fronsdal, In Essays On Supersymmetry, edited by C. Fronsdal (UCLA, Los Angeles 1985), p. 163; see book index.

[52] O. A. Gelfond and M. A. Vasiliev, Symmetries of higherspin current interactions in four dimensions, Teor. Mat. Fiz. 187, 401 (2016); Theor. Math. Phys. 187, 797 (2016).

[53] S. Deser, E. Joung, and A. Waldron, Partial masslessness and conformal gravity, J. Phys. A 46, 214019 (2013).

[54] K. Hinterbichler and A. Joyce, Manifest duality for partially massless higher spins, J. High Energy Phys. 09 (2016) 141.

[55] S. Deser and A. Waldron, PM = EM: Partially massless duality invariance, Phys. Rev. D 87, 087702 (2013). 